\title{
Estresse Ocupacional em Profissionais da Saúde que Atuam no Serviço de Atendimento Móvel de Urgência de Cajazeiras - PB
}

\section{Ocupational Stress Among Health Professionals Working in an Emergency Mobile Care Service in the City of Cajazeiras - PB}

\author{
MARIA SORAYA PEREIRA FRANCO ADRIANO \\ MÔNICA RAFAELA DE ALMEIDA ${ }^{2}$ \\ PÂMELA PERONICO LEITE RAMALHO ${ }^{3}$ \\ ILUSKA PINTO DA COSTA ${ }^{4}$ \\ AISSA ROMINA SILVADO NASCIMENTO ${ }^{4}$ \\ JANAÍNE CHIARA OLIVEIRA MORAES ${ }^{5}$
}

\section{RESUMO}

Objetivo: Avaliar o nível de estresse ocupacional da equipe de saúde atuante no Serviço de Atendimento Móvel de Urgência. Materiais e métodos: Trata-se de um estudo descritivo com abordagem quantitativa, realizado com 24 profissionais do SAMU do município de Cajazeiras - PB, no qual utilizou-se como instrumento de coleta o Inventário de Sintomas de Stress para Adultos de Lipp. Resultados: 16,7\% dos profissionais são acometidos pelo estresse no ambiente de trabalho, onde destes, todos se encontram na fase de Resistência. Quanto à sintomatologia consequente ao estresse, os homens desencadearam predominantemente sintomas psicológicos, enquanto as mulheres desenvolveram alterações nos aspectos físicos e psicológicos, simultaneamente. Conclusão: Vislumbra-se que as atividades laborais exercidas pelos profissionais do SAMU geram reações positivas para o estresse, em que os aspectos mais afetados estão ligados ao psicológico e à sobrecarga física, condições que se não prevenidas, concorrem para prejuízos na saúde do trabalhador, na produtividade, na organização do serviço e especialmente na assistência prestada à população.

\section{DESCRITORES}

Serviços Médicos de Emergência. Esgotamento profissional. Ambiente de trabalho. Doenças profissionais.

\begin{abstract}
Objective: To assess the level of occupational stress among health professionals working in an Emergency Mobile Care Service (SAMU). Material and Methods: This was a descriptive study with a quantitative approach including 24 SAMU professionals in the city of Cajazeiras, PB. The Inventory of Stress Symptoms for Adults by Lipp was used for data collection. Results: $16.7 \%$ of the professionals were found to be affected by stress in the workplace. Of these, all were experiencing the resistance phase. Among the symptoms resulting from stress, men demonstrated predominantly psychological symptoms, while women developed abnormal physical and psychological conditions simultaneously. Conclusion: The working activities performed by SAMU professionals favor positive reactions to stress, in which the most affected aspects are linked to psychological and physical overload conditions. If not prevented, these factors may impair the worker's health, productivity , organization of the service and, in particular, the assistance provided to the population.
\end{abstract}

\section{DESCRIPTORS}

Emergency Medical Services. Burnout. Desktop. Professional diseases.

\footnotetext{
Docente Doutora da Universidade Federal da Paraíba (UFPB). João Pessoa. Paraíba. Brasil.

Psicóloga da Universidade Federal Rural do Semi-Árido (UFERSA). Mossoró. Rio Grande do Norte. Brasil.

Enfermeira graduada pela Universidade Federal de Campina Grande. Cajazeiras. Paraíba. Brasil.

Docente da Universidade Federal de Campina Grande (UFCG). Cajazeiras. Paraíba. Brasil.

5 Docente Mestre da Universidade Federal de Campina Grande (UFCG). Cajazeiras. Paraíba. Brasil.
} 
$\mathrm{A}$ s transformações que estão ocorrendo no cotidiano dos trabalhadores, ao longo do tempo, vêm desenhando um novo modo de vida e definindo diferentes padrões biopsicossociais, o que causa forte influência sobre a produtividade do trabalho. $\mathrm{O}$ estresse ocupacional pode ser entendido como um processo de relação entre as atividades laborais, saúde e doença. Algumas ocupações, no entanto, se caracterizam como mais estressantes que outras, porém, o fator determinante é influenciado pela subjetividade individual $^{1}$. Diversas pesquisas têm apontado que o estresse ocupacional é uma condição que acomete, com maior frequência, os profissionais da saúde, trazendo prejuízos não só à saúde, como também à qualidade da assistência prestada pelos mesmos ${ }^{2-4}$.

No que se refere ao trabalho na área da saúde, as necessidades imediatas, agudas ou de urgência da população são pontos de pressão que exigem dos profissionais respostas rápidas, de modo que o atendimento aos pacientes seja de tal maneira que permita o mínimo de consequências, no que tange à negligência de ações, a erros assistenciais e a exposição a condições agravantes ${ }^{5}$.

Com base nessa percepção, foi instituído o atendimento pré-hospitalar, que corresponde aos cuidados estabelecidos na cena do acidente e no transporte da vítima até o ambiente hospitalar. Através da Portaria $n^{\circ}$ 1864/2003, o Ministério da Saúde implanta o Serviço de Atendimento Móvel de Urgência (SAMU) no Brasil. Este surge enquanto forma de assistir às demandas de urgência, seja no trabalho, no domicilio, em vias públicas ou aonde o paciente vier a precisar. $\mathrm{O}$ SAMU conta com equipes de Suporte Básico e Suporte Avançado de Vida, funcionando como uma regulação médica e elemento ordenador e orientador da atenção pré-hospitalar. A recepção, análise e priorização do atendimento acontecem através de uma Central de Regulação Médica que estabelece uma porta aberta de comunicação do público com o sistema de saúde, sendo o pedido de socorro acolhido no menor intervalo de tempo possível ${ }^{5}$.

Com a implementação dessa modalidade de atendimento, houve redução significativa do número de óbitos, do tempo de internação hospitalar e das sequelas decorrentes da falta de socorro precoce. O serviço atua 24 horas por dia, sendo constituído por uma equipe multidisciplinar composta por médicos, enfermeiros, técnicos de enfermagem e motoristas que atendem às urgências de natureza traumática, clínica, pediátrica, cirúrgica, gineco-obstétrica e de saúde mental $^{6}$.

Vários são os fatores que podem levar o profissional atuante no SAMU ao estresse ocupacional.
Dentre eles destacam-se, a incorporação de atividades cada vez mais técnicas e especializadas, a tomada de decisões sobre forte pressão e a resolução de problemas. Somam-se a isto as peculiaridades do atendimento às urgências, em que o profissional lida direta e indiretamente com questões ligadas à morte ${ }^{3}$.

Devido às características mencionadas, os profissionais sofrem constante tensão psicológica em detrimento da intensidade dos casos com que se deparam em seu dia-a-dia. Estes trabalhadores perpassam por situações limítrofes, muitas vezes, com risco de comprometimento da sua própria saúde. Todas essas variáveis contribuem para o desgaste da equipe de saúde, podendo acarretar um elevado grau de estresse ocupacional. A assistência no serviço de urgência exige, portanto, conhecimento técnicocientífico, segurança e autocontrole ${ }^{5,7}$.

Mediante o exposto, é incitante a reflexão: de que forma as atividades laborais geram estresse nos profissionais do Serviço de Atendimento Móvel de Urgência? O presente estudo torna-se valoroso a partir do momento que busca conhecer os desencadeantes do estresse no trabalho, ressaltando o impacto que ele pode causar na vida dos profissionais. Em vista à relevância desta problemática, objetivou-se avaliar o nível de estresse ocupacional da equipe de saúde atuante no SAMU. Além disso, delinearam-se como objetivos específicos, identificar as fases de estresse em que se encontram os profissionais deste serviço, bem como verificar a frequência dos sintomas físicos e psicológicos apresentados por estes.

\section{MATERIAIS E MÉTODOS}

Trata-se de um estudo transversal, quantitativo, com procedimento comparativo e descritivo, realizado com uma equipe multiprofissional da base Regional do Serviço de Atendimento Móvel a Urgências (SAMU) do município de Cajazeiras - PB. Utilizou-se uma amostragem por conveniência nãoprobabilística, sendo a amostra constituída por 24 profissionais de saúde. Para seleção das participantes utilizou-se como critérios de inclusão: ser membro em exercício da equipe de saúde referida, estar presente no local no momento da coleta de dados e aceitar participar do estudo mediante consentimento livre e esclarecido.

Inicialmente foram realizadas visitas à instituição, no intuito de apresentar a proposta de trabalho. Em seguida foi realizado um pré-teste dos instrumentos, com seis profissionais do serviço, a fim de averiguar os termos e a existência de vieses. Decorrido essa etapa inicial, a coleta dos dados se deu entre os 
meses de abril e maio de 2011, utilizando-se o Inventário de Sintomas de Stress para Adultos (ISSL).

O ISSL é baseado em um modelo quadrifásico de estresse, proposto por Lipp e Guevara em 2000. Esse questionário é de fácil aplicação e visa identificar de modo objetivo a sintomatologia apresentada, somática ou psicológica, bem como a fase de estresse em que o indivíduo se encontra. Esse instrumento é composto por três quadros referentes às fases de estresse: alerta, resistência (e quase-exaustão) e exaustão. Os sintomas físicos (F) e psicológicos (P) que aparecem em cada quadro são os típicos de cada fase.

Os dados foram analisados e interpretados por uma Psicóloga, tendo como referência as normas do Manual do ISSL, conforme preconiza a resolução do Conselho Federal de Psicologia (CFP) N. ${ }^{\circ} 25 / 2001$, que define os Testes Psicológicos como instrumentos de avaliação de uso privativo do psicólogo? .

O estudo seguiu todas as observâncias éticas como preza a Resolução 196/96 do Código de Ética em Pesquisa com humanos - que garante ao participante o total anonimato e a liberdade de recusa ou retirada do seu consentimento em qualquer fase do estudo, sendo a pesquisa previamente submetida à apreciação pelo Comitê de Ética em Pesquisa da Universidade Estadual da Paraíba e aprovada por este comitê sob Parecer $n^{\circ}$ 00860133000/11. O mesmo não obteve nenhum tipo de financiamento, não apresentando também conflitos de interesses.

\section{RESULTADOS}

Participaram da pesquisa 24 profissionais de saúde, sendo quatro médicos, oito enfermeiros e 12 técnicos de enfermagem. Em relação ao sexo, percebeuse uma predominância do feminino $(58,3 \%, \mathrm{n}=14)$ sobre o masculino $(41,7 \%, n=10)$. Quanto à faixa etária, observa-se que $62,5 \%(n=15)$ desses trabalhadores tem idade entre 20 e 30 anos, $25 \%(n=6)$ tem idade entre 31 e 40 anos, e 12,5\% $(n=3)$ destes corresponde à faixa etária de 41 a 50 anos. Quanto à escolaridade, $50 \%(n=12)$ dos profissionais possui nível médio, o que pode estar relacionado ao maior número de técnicos de enfermagem na amostra.

Quanto ao tempo de atuação no serviço, 83,3\% $(n=22)$ dos participantes trabalha há menos de um ano na instituição, 8,3\% $(n=1)$ entre um e cinco anos e $8,3 \%$ $(n=1)$ entre seis e dez anos, evidenciando que a amostra constitui-se de trabalhadores com pouca experiência na área. A carga horária de trabalho semanal referida, somando-se todos os vínculos empregatícios, foi de mais de mais de 40 horas de dedicação para $62,5 \%(n=15)$ dos profissionais. Porém, no que concerne à remuneração, revelou-se uma desigualdade salarial, uma vez que, 70,8\% $(n=16)$ dos profissionais perfaz uma renda estimada entre um e quatro salários mínimos, enquanto que $12,5 \%(n=3)$ ganha entre cinco e oito salários e $16,7 \%(\mathrm{n}=5)$ obtém o correspondente a mais de 12 salários mínimos (nessa porcentagem estão incluídos os médicos). Durante a pesquisa identificouse ainda que $12,5 \%(n=3)$ dos participantes fazem uso de cigarro e $29,2 \%(n=8)$ consume algum tipo de bebida alcoólica.

\section{O nível de estresse nos profissionais da saúde do SAMU}

Os dados abaixo demonstram que $62,5 \%(n=15)$ dos trabalhadores não apresentaram estresse ocupacional, o que em suma, não condiz com a realidade vivenciada pelos profissionais de saúde, principalmente com aqueles atuantes nos serviços de urgência e emergência. Pode-se levar em consideração para esse resultado que um número significativo de participantes $(20,8 \%, n=4)$ não respondeu ao inventário, conforme mostra a tabela 1 .

As estatísticas oficiais e não oficiais apontam que o número de trabalhadores acometidos por transtornos mentais e de comportamento, associados ao trabalho, vem crescendo a cada dia. Dentro desse universo, se encontra os trabalhadores da saúde, que vêm adoecendo cada vez mais em decorrência das atividades laborais ${ }^{2,11}$. Apesar dos dados obtidos não se relacionarem fidedignamente com a literatura, ainda assim, o estudo aponta que uma parcela significativa de profissionais do atendimento pré-hospitalar (16,7\%, $\mathrm{n}=5$ ) são acometidos pelo estresse no ambiente de trabalho.

A tabela 2 revela que todos os participantes que apresentaram estresse ocupacional $(16,7 \%, n=5)$ encontram-se na fase de Resistência, que se caracteriza pela irritabilidade, insônia, mudanças no humor, diminuição da libido e atrofia de algumas estruturas relacionadas à produção de células sanguíneas.

Em relação à distribuição dos participantes de acordo com a sintomatologia consequente do estresse, nota-se que os homens desencadeiam predominantemente sintomas psicológicos, enquanto que as mulheres desenvolvem sintomas físicos e psicológicos simultaneamente, conforme se pode verificar na tabela 3. 


\begin{tabular}{|c|c|c|c|}
\hline Sexo & $\begin{array}{c}\text { Sem Estresse } \\
\mathrm{N}(\%)\end{array}$ & $\begin{array}{c}\text { Com Estresse } \\
\mathrm{N}(\%)\end{array}$ & $\begin{array}{c}\text { Não Respondeu } \\
N(\%)\end{array}$ \\
\hline Masculino & $5(16,7)$ & $2(4,2)$ & $3(12,5)$ \\
\hline Feminino & $10(45,8)$ & $3(12,5)$ & $1(8,3)$ \\
\hline Total & $15(62,5)$ & $5(16,7)$ & $4(20,8)$ \\
\hline
\end{tabular}

\begin{tabular}{|c|c|c|c|}
\hline Fases do estresse & $\begin{array}{c}\text { Masculino } \\
\text { N (\%) }\end{array}$ & $\begin{array}{c}\text { Feminino } \\
\mathbf{N}(\%)\end{array}$ & Total N (\%) \\
\hline Alerta & - & - & - \\
\hline Resistência & $2(4,2)$ & $3(12,5)$ & $5(16,7)$ \\
\hline Quase-exaustão & - & - & - \\
\hline Exaustão & - & - & - \\
\hline
\end{tabular}

\begin{tabular}{|c|c|c|}
\hline \multicolumn{3}{|c|}{$\begin{array}{l}\text { Tabela 3. Distribuição dos participantes por } \\
\text { sintomatologia predominante. Cajazeiras - PB. Brasil. } \\
\text { 2011; }(n=5)\end{array}$} \\
\hline Sintomatologia & $\begin{array}{c}\text { Masculino } \\
\mathrm{N}(\%)\end{array}$ & $\begin{array}{c}\text { Feminino } \\
\mathrm{N}(\%)\end{array}$ \\
\hline Física & - & - \\
\hline Psicológica & $2(4,2)$ & - \\
\hline Física e Psicológica & - & $3(12,5)$ \\
\hline Total & $2(4,2)$ & $3(12,5)$ \\
\hline
\end{tabular}

\section{DISCUSSÃO}

Os resultados obtidos com a presente pesquisa indicam uma alta porcentagem de trabalhadores que não apresentaram estresse. $\mathrm{O}$ não acometimento desses pode estar relacionado, principalmente, a fatores como: baixa idade, já que $62,5 \%$ dos participantes estão inseridos na faixa etária entre 20 e 30 anos, além de pouco tempo de trabalho no serviço. Assim, o fato de serem jovens pode estar contribuindo para que o organismo resista com mais vigor aos agentes estressores, obtendo, dessa forma, maior disposição para realizar suas atividades. Já a menor experiência profissional possibilita o aumento do interesse pelo trabalho, uma vez que, existe nessa fase, um enorme desejo de aprender e aperfeiçoar o mais rápido possível suas técnicas e habilidades ${ }^{12}$.

Outro fator que poderia explicar o resultado mencionado acima pode estar relacionado ao medo de perseguição, como foi observado através de alguns comentários feitos pelos próprios participantes durante a coleta dos dados. Esse medo se fundamenta na origem da ocupação dos cargos, uma vez que, naquela instituição não existem profissionais efetivos, sendo o critério de seleção para as vagas realizada mediante indicação política. Portanto, acredita-se que alguns participantes omitiram suas respostas no intuito de não comprometer seu emprego.

Quanto aos 16,7\% dos profissionais que apresentaram estresse ocupacional, pode-se associá-lo à sobrecarga de trabalho, uma vez que, estes possuem uma carga horária de trabalho exaustiva (superior a 40 horas semanais). Os dados revelam que esses trabalhadores possuem mais de um vínculo empregatício, já que os salários, muitas vezes, são baixos e não satisfazem suas necessidades. É possível, também, correlacionar o estresse evidenciado por estes trabalhadores à complexidade das tarefas executadas, bem como ao comprometimento e responsabilidades 
exigidas nesse tipo de assistência. Atribui-se, ainda, o fato de estarem em contato direto e constante com a dor e o sofrimento das pessoas ${ }^{13}$.

Ressalta-se também uma predominância das mulheres sobre os homens quando se trata do estresse no trabalho. A princípio, pode estar relacionado à composição da amostra, pois $66,7 \%$ dos participantes é do sexo feminino. Em um segundo plano, pode-se fazer uma análise no sentido de se considerar a dupla ou até tripla jornada de trabalho enfrentada por essas profissionais. Muitas dessas mulheres têm um ou mais empregos e ainda precisam cuidar do lar e dos filhos, quando possuem.

O estresse é uma reação, com componentes físicos e emocionais, que o organismo apresenta diante de qualquer situação que represente um desafio maior. Pode ter sentido positivo ou negativo. Ele é positivo quando se encontra em sua fase inicial (alerta), em que o indivíduo apresenta força e vigor para lutar ou fugir das situações mais difíceis. Caso o estresse persista, o indivíduo se cansa em excesso, entrando na fase de resistência, caracterizada por sensação de desgaste generalizado, problemas com a memória e dúvidas quanto a si próprio. A fase seguinte é a de quaseexaustão, nela a tensão excede o limite do gerenciável, a resistência física e emocional começa a se quebrar e doenças começam a surgir. E, por fim, vem a fase de exaustão, considerada a mais negativa. É quando ocorre um desequilíbrio interior muito grande, o indivíduo não consegue mais se concentrar, trabalhar, nem tomar decisões sensatas. Começam, então, a surgir as doenças mais graves. Retomar o equilíbrio do organismo tornase uma tarefa muito difícil nessa fase $\mathrm{e}^{7,10}$.

Todos os participantes da pesquisa que apresentaram estresse (16,7\%) encontram-se na fase de Resistência. Assim, entende-se que estes trabalhadores já estão sob ação de agentes estressores há algum tempo, os quais podem estar relacionados não somente à organização de trabalho como também a fatores de ordem social. Essa fase funciona como uma ponte de transição entre o estresse positivo e o negativo, portanto, é importante que os fatores estressores sejam identificados o mais rápido possível, para tentar impedir que o quadro de estresse evolua, não permitindo que o indivíduo atinja as fases mais negativas do estresse (Quase-exaustão e Exaustão) $^{8}$.

Em relação à sintomatologia, percebe-se que houve uma predominância dos sintomas físicos e psicológicos, apresentados concomitantemente, sobre os sintomas apenas psicológicos. Fato justificado devido a esses indivíduos se encontrarem na fase de Resistência. Portanto, os participantes mostraram-se mais vulneráveis emocionalmente do que fisicamente.

Dessa maneira, esses profissionais devem receber especial atenção, por meio de programas sistemáticos de educação que abordem maneiras de identificação de fatores de risco para o estresse, bem como, desenvolvimento de estratégias de enfrentamento da doença ${ }^{1,14}$. As primeiras incluem diálogo, empatia, ajuda mútua e resolução de conflitos. As estratégias fora do trabalho incluem lazer, meditação, relaxamento, estar com a família e valorizar a vida. O gerenciamento do estresse ocupacional pode repercutir em melhora do desempenho dos profissionais, com preservação da saúde e ampliação da qualidade da assistência ${ }^{15}$.

Programas de manejo podem ser focados na organização e/ou no trabalhador. Intervenções focadas na organização são voltadas para a modificação de estressores do ambiente de trabalho, podendo incluir transformações na estrutura, condições laborais, treinamento, participação e autonomia de decisões, bem como boas relações interpessoais. Já as intervenções focadas no indivíduo almejam reduzir o impacto de riscos já existentes, através do desenvolvimento de um adequado repertório de medidas de enfrentamento individuais ${ }^{12}$. Caso a equipe tenha espaço para discussão, os estressores terão sua ação negativa reduzida, diminuindo, assim, os impactos na saúde do indivíduo. Nessa perspectiva, acredita-se que a mudança de atitude permite uma melhor forma de lidar com os fatores estressantes presentes no ambiente de trabalho, principalmente, quando há o investimento em relações humanas saudáveis.

\section{CONCLUSÃO}

Diante o apresentado, vislumbra-se que as atividades laborais exercidas pelos profissionais do SAMU geram reações positivas para o estresse, em que os aspectos mais afetados estão ligados ao psicológico e à sobrecarga física, condições que se não prevenidas, concorrem para prejuízos na saúde do trabalhador, na produtividade, na organização do serviço e especialmente na assistência prestada à população. 


\section{REFERÊNCIAS}

1. Maia EC, Miranda MDC, Caetano JA, Carvalho ZMF, Santos MCL, Caldini LN. Avaliação do nível de estresse de equipe de enfermagem de serviço de atendimento móvel de urgência. R. pesq.: cuid. fundam. Online. 2012:4(4):306068.

2. Camelo SHH, Angerami ELS. Sintomas de estresse nos trabalhadores atuantes em cinco núcleos de saúde da família. Ribeirão Preto: Revista Latino-Americana de Enfermagem, 2004;12(1):14-21.

3. Carvalho L, Malagris LEN. Avaliação do nível de stress em profissionais da saúde. Rio de Janeiro: Estudos e Pesquisas em Psicologia, 2007;7(3):570-82.

4. Cavalheiro A M, Junior DFM, Lopes AC. Estresse de enfermeiros com atuação em unidade de terapia intensiva. Ribeirão Preto: Revista Latino-Americana de Enfermagem, 2008;16(1):29-35.

5. Campos RM. Satisfação da equipe de enfermagem do Serviço de Atendimento Móvel às Urgências (SAMU) no ambiente de trabalho. 128 f. Dissertação (Mestrado em Ciências da Saúde) - Universidade Federal do Rio Grande do Norte, Natal - RN, 2005.

6. Martins CCF, Vieira NA, Morais FRR. O desgaste relacionado ao trabalho na ótica dos enfermeiros de atendimento pré-hospitalar. R. pesq.: Cuid. Fundam. Online. 2011:3(2):2024-32.

7. Brasil, Ministério da Saúde. Política Nacional de Atenção às urgências. Brasília, 2003.

8. Lipp MEN. Manual do Inventário de Sintomas de Stress para Adultos de Lipp (ISSL). 2 ed. São Paulo: Casa do Psicólogo, 2000.
9. Conselho Federal de Psicologia. Resolução $n^{\circ} 25,30$ de novembro de 2001. Define teste psicológico como método de avaliação privativo do psicólogo e regulamenta sua elaboração, comercialização e uso. Brasília, DF. 2001.

10. Stumm EMF, Ribeiro G, Kirchner RM, Loro MM, Rosaneli CLSP. Avaliação da saúde e qualidade de vida: profissionais de um SAMU. Cogitare Enferm. 2009;14(4):620-7.

11. Azambuja EP, Kerber NPC, Kirchhof AL. A saúde do trabalhador na concepção de acadêmicos de enfermagem. São Paulo: Revista da Escola de Enfermagem da USP, 2007;41(3): 355-62.

12. Murofuse NT, Abranches SS, Napoleão AA. Reflexões sobre estresse e Burnout e a relação com a enfermagem. Ribeirão Preto: Revista. Latino-Americana de Enfermagem, 2005;13(2):255-61.

13. Andrade ML, Caetano JA, Soares E. Percepção das enfermeiras sobre a unidade de emergência. Fortaleza: Revista RENÉ, 2000;1(1):91-97.

14. Murta SG. Programas de manejo de estresse ocupacional: uma revisão sistemática da literatura. São Paulo: Revista Brasileira de Terapia Comportamental e Cognitiva, 2005;7(2):159-77.

15. Silveira MM, Stumm EMF, Kirchner RM. Estressores e coping: enfermeiros de uma unidade de emergência hospitalar. Rev. Eletr. Enf. [Internet]. 2009; 11(4):894-903.

\section{Correspondência}

Nome: Janaíne Chiara Oliveira Moraes

Endereço: Rua Fausto Rolim, 156; Bairro: Capoeiras.

CEP: 58900-000.

Cajazeiras - Paraíba - Brasil.

E-mail: janainechiara@hotmail.com 